\title{
Auditory priming of pseudo-suffixed words
}

Ava Creemers, Nattanun Chanchaochai, David Embick

Department of Linguistics, University of Pennsylvania, USA

https://doi.org/10.36505/ExLing-2019/10/0016/000378

\begin{abstract}
We present results from two auditory experiments that examine priming effects for pseudo-derived pairs of words (e.g., corner-corn), as compared to morphologically, phonologically, and semantically related pairs. Previous work shows facilitation for pseudo-derived pairs in a visual masked priming paradigm; our Experiment 1 presents the novel finding that these effects are also found in auditory priming, with no consistent difference between pseudo-derived and phonologically-related pairs. Experiment 2 introduces an intervener between primes and targets to further probe the nature of the facilitation in pseudo-derived pairs. Implications for theories of morphological processing and spoken-word recognition are discussed.
\end{abstract}

Key words: auditory word recognition, morphological priming, pseudo-suffixation

\section{Introduction}

Visual masked priming experiments have focused on different relations between words and their embedded parts, including prime-target pairs that are morphologically and semantically related (teacher-teach), and pseudo-derived pairs like corner-corn. In the latter, corner occurs with an existing stem and suffix but is not etymologically or semantically related to corn. Pseudo-derived words have been used to argue for orthography-driven decomposition of words (Rastle et al. 2004, Rastle and Davis 2008, i.a.), for morphological decomposition independent of semantic transparency (Marslen-Wilson et al. 2008, i.a.), and for both sides in the debate between form-then-meaning (Beyersmann et al. 2016, Longtin et al. 2003) and form-and-meaning (Feldman et al. 2009; 2015, Milin et al. 2017) approaches.

Some questions are unanswered: Are decomposition effects for pseudoderived items restricted to orthography (cf. Rastle, Davis 2008), or can they also be found in the auditory modality? And, are effects for pseudo-derived words, which do not actually share a morphological relation with their stem, morphological in nature, or merely form-driven? To investigate these issues, we present two primed lexical decision experiments with auditorily presented primes and targets. Experiment 1 shows that priming effects for pseudoderived words can be obtained in the auditory modality. Experiment 2 investigates the nature of these effects by including an intervening item between primes and targets, based on the idea that phonological facilitation decays more rapidly than morphological facilitation.

ExLing 2019: Proceedings of 10 $10^{\text {th }}$ International Conference of Experimental Linguistics, 25-27 September 2019, Lisbon, Portugal 


\section{Experiments 1 and 2 Methods}

Conditions include prime-target pairs that are morphologically and semantically related (MS: creamy-cream, treatment-treat), pseudo-morphologically related ((pseudo-)M: belly-bell, pigment-pig), purely phonologically related with no (pseudo-)suffix (Ph: dogma-dog, pillow-pill), and semantically related (S: garbagetrash, painting-art). The pseudo-M and MS conditions differ as minimally as possible, with different suffixes appearing equally often in these conditions. $\mathrm{M}$ pairs that share a remote or archaic meaning (e.g., archer) were excluded. Targets are orthographic and phonological sub-strings of their prime. The meaning of the prime in MS pairs can always be derived from the meaning of the stem, as evidenced by high pairwise Latent Semantic Analysis (LSA) measures. S primes were also selected based on high LSA measures with the target. Targets are monosyllabic; primes are disyllabic with main stress on the first syllable.

In total, we included 20 prime-target pairs per condition. Unrelated primes were selected for each pair. Subject saw 80 critical pairs of which half were unrelated. Subjects were allocated to one of two lists, so that they saw each target only once. For the purpose of the lexical decision task, 100 filler words and 260 non-words were included. Finally, to investigate the time-course with which different types of linguistic information become available, we manipulated the Inter-Stimulus Interval (ISI) as a between-subject factor in Experiment 1. We included ISIs of $200-400 \mathrm{~ms}$ (short-ISI), $600-800 \mathrm{~ms}$ (medium-ISI), and 1000-12000ms (long-ISI). In Experiment 2, we used a lag of 1 intervening item and a medium-ISI between prime and target to investigate the nature of the effects in the $\mathrm{M}$ condition, aiming to tease apart phonological and morphological effects.

Stimuli were recorded by an adult native speaker of American English. A lexical decision task was implemented in PsychoPy2. Participants were native speakers of English (122 in Experiment 1; 80 in Experiment 2), who received course credit as compensation for participation. They were instructed that they would hear existing and non-existing words and had to make a lexical decision to each word as quickly and accurately as possible.

\section{Results Experiment 1}

Responses were coded for response type and response time (RT). Differences in duration of the sound files were included in the model. Incorrect responses to primes and targets were discarded, and one subject was excluded due to low accuracy. We combine a-priori data trimming with model criticism, and analyze log-transformed RTs to targets with linear mixed-effects models.

The results are illustrated in Figure 1. Collapsing the different ISIs used, significant priming effects are found in all conditions $(p<0.001$ for $\mathrm{MS}, \mathrm{M}, \mathrm{Ph}$, $S)$, which also holds for each individual ISI-version. The model indicates a larger effect in the MS condition compared to the M $(p<0.001), \mathrm{Ph}(p<$ 
0.001), and $\mathrm{S}(p<0.001)$ conditions, which again holds regardless of ISI-group. No difference is found between $\mathrm{M}$ and $\mathrm{Ph}(p=0.956)$ in the combined data, or at a short-ISI $(p=0.617)$ and medium-ISI $(p=0.059)$. However, a significantly greater priming effect is found for the $\mathrm{Ph}$ condition compared to $\mathrm{M}$ at a LongISI $(p=0.028)$.

The results show that priming effects for pseudo-derived (M) pairs can be obtained in the auditory modality. Experiment 2 investigates whether the effect in the $\mathrm{M}$ condition is morphological or phonological in nature.
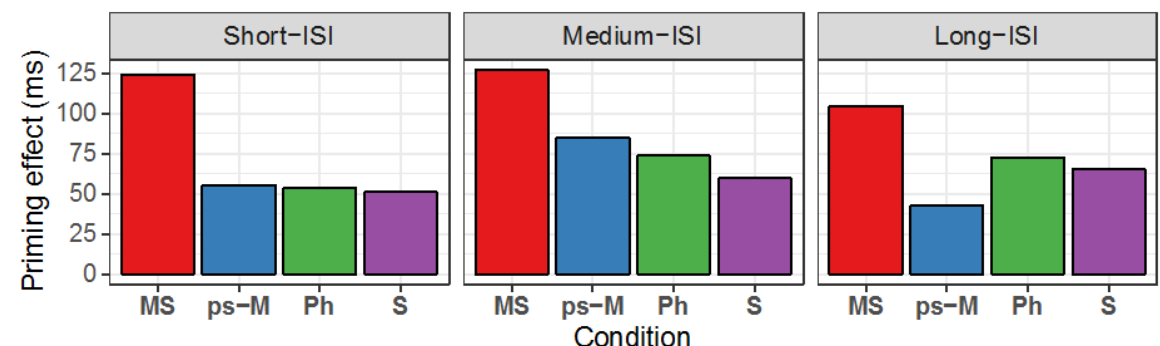

Figure 1. Priming effects (in $\mathrm{ms}$ ) in Experiment 1. Priming effects reflect the difference between the RT to a target preceded by a related versus unrelated prime.

\section{Results Experiment 2}

We analyzed log-transformed RTs with lme models. The results at Immediate Distance replicate the results at a medium-ISI in Experiment 1. The difference in priming between $\mathrm{M}$ and $\mathrm{Ph}$ was significant $(p=0.006)$. With One Intervener, we find significant priming only for MS $(p<0.001)$, but not for M $(p=0.312)$, $\mathrm{Ph}(p=0.051)$, and $\mathrm{S}(p=0.110)$. No difference is found between the priming effects in MS and $\mathrm{M}(p=0.078)$, and $\mathrm{M}$ and $\mathrm{Ph}(p=0.517)$.

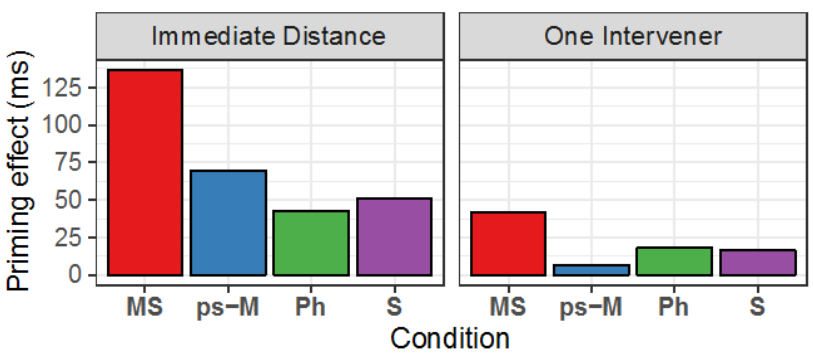

Figure 2. Priming effects (in ms) in Experiment 2. 


\section{General discussion}

The results show priming effects in all conditions, including the pseudo-M condition. This suggests that pseudo-derived words prime their 'stem' in the auditory modality, and that priming effects for pseudo-derived word pairs are therefore not merely an artefact of orthographic representations. However, the effect for pseudo-M is smaller than in the MS condition (in line with Feldman et al. 2015), and not consistently different from the phonological effect in $\mathrm{Ph}$ (in line with Milin et al. 2017). The lack of priming for pseudo-M with an intervener suggests that the effect is form-driven, and not morphological. In addition, our results have implications for theories of auditory word recognition. Specifically, the results in our $\mathrm{Ph}$ condition suggests that cohort competitors (e.g., dog to dogma, and pill to pillow) may stay activated longer than previously thought, which would explain the priming effects in the pseudoderived condition as well.

\section{Acknowledgements}

Many thanks are due to Meredith Tamminga, Robert J. Wilder, Amy Goodwin Davies, Hezekiah Akiva Bacovcin, and Kobey Shwayder.

\section{References}

Beyersmann, E., Ziegler, J.C., Castles, A., Coltheart, M., Kezilas, Y., Grainger, J. 2016. Morpho-orthographic segmentation without semantics. Psychonomic Bulletin and Review, 23(2), 533-539.

Feldman, L.B., O’Connor, P.A., del Prado Martín, F.M. 2009. Early morphological processing is morphosemantic and not simply morpho-orthographic. Psychonomic Bulletin and Review, 16(4), 684-691

Feldman, L.B., Milin, P., Cho, K.W., Moscoso del Prado Martín, F., O’Connor, P. A. 2015. Must analysis of meaning follow analysis of form? A time course analysis. Frontiers in human neuroscience, 9, 111.

Longtin, C.-M., Segui, J., Halle, P. 2003. Morphological priming without morphological relationship. Language and Cognitive Processes, 18(3), 313-334.

Marslen-Wilson, W. D., Bozic, M., Randall, B. 2008. Early decomposition in visual word recognition: Dissociating morphology, form, and meaning. Language and Cognitive Processes, 23(3), 394-421.

Milin, P., Feldman, L.B., Ramscar, M., Hendrix, P., Baayen, R.H. 2017. Discrimination in lexical decision. PloS one, 12(2), e0171935.

Rastle, K., Davis, M.H. 2008. Morphological decomposition based on the analysis of orthography. Language and Cognitive Processes, 23(7-8), 942-971.

Rastle, K., Davis, M.H., New, B. 2004. The broth in my brother's brothel: Morphoorthographic segmentation in visual word recognition. Psychonomic Bulletin and Review, 11(6), 1090-1098. 